\title{
Existence of periodic solutions for a type of linear difference equations with distributed delay
}

\author{
Jehad O Alzabut
}

Correspondence: jalzabut@psu.edu. sa

Department of Mathematics and Physical Sciences, Prince Sultan University, P. O. Box 66833, 11586 Riyadh, Saudi Arabia

\section{Abstract}

By employing primary algebraic techniques, we establish a necessary and sufficient condition for the existence of periodic solutions for a type of linear difference equations with distributed delay of the form

$\Delta x(n)=\sum_{k=-d}^{0} \Delta_{k} \zeta(n+1, k-1) x(n+k-1), \quad n \geq 1$. (*) $^{*}$

Our approach is based on constructing an adjoint equation for $\left(^{*}\right)$ and proving that $\left.{ }^{*}\right)$ and its adjoint equation have the same number of linearly independent periodic solutions.

AMS Subject Classification: 39A11.

Keywords: difference equations, periodic solutions, adjoint, distributed delay

\section{Introduction}

Let $\mathbb{N}, \mathbb{Z}, \mathbb{R}$ be the sets of natural, integer and real numbers, respectively. By $\mathbb{R}^{m}$, we denote the $m$-dimensional Euclidean space with elements $x=\operatorname{col}\left(x_{1}, x_{2}, \ldots, x_{m}\right)$.

It is well known that the nonhomogeneous linear equation $x^{\prime}(t)=A(t) x(t)+f(t)$ has periodic solutions if and only if

$$
\int_{0}^{\omega} y^{T}(t) f(t) \mathrm{d} t=0
$$

for all periodic solutions $y(t)$ of period $\omega$ of the adjoint equation $y^{\prime}(t)=-A^{T}(t) y(t)$, where $A \in C\left(\mathbb{R}, \mathbb{R}^{m \times m}\right)$ and $f \in C\left(\mathbb{R}, \mathbb{R}^{m}\right)$ are periodic functions of period $\omega$; see for instance [1]. By " $T$ ", we mean the transposition.

In his remarkable monograph [2], Halanay extended the above result to linear delay differential equations of the form

$$
x^{\prime}(t)=A(t) x(t)+B(t) x(t-\tau)+f(t), \quad t>0,
$$

where $A, B \in C\left(\mathbb{R}, \mathbb{R}^{m \times m}\right)$ and $f \in C\left(\mathbb{R}, \mathbb{R}^{m}\right)$ are periodic functions of period $\omega$ and $\tau$ $>0$ is a fixed real number. It was shown that the required condition involves the same integral (1). Indeed, Halanay proved that Equation (2) has periodic solutions if and only if (1) holds for all periodic solutions $y(t)$ of period $\omega$ of the adjoint equation

$$
\gamma^{\prime}(t)=-A^{T}(t) y(t)-B^{T}(t+\tau) y(t+\tau),
$$

(c) 2012 Alzabut; licensee Springer. This is an Open Access article distributed under the terms of the Creative Commons Attribution License (http://creativecommons.org/licenses/by/2.0), which permits unrestricted use, distribution, and reproduction in any medium, provided the original work is properly cited. 
which is constructed with respect to the function

$$
<y(t), x(t)>=y^{T}(t) x(t)+\int_{t}^{t+\tau} y^{T}(s) B(s) x(s-\tau) \mathrm{d} s .
$$

The same problem has been investigated for linear impulsive delay differential equations [3,4]. The discrete analog of the above mentioned result has been recently studied in [5]. We suggest the reader to consult [6-10] for more results regarding existence of periodic solutions for difference equations.

The purpose of this article is to establish a necessary and sufficient condition for the existence of periodic solutions for a type of linear difference equation with distributed delay of the form

$$
\Delta x(n)=\sum_{k=-d}^{0} \Delta_{k} \zeta(n+1, k-1) x(n+k-1), \quad n \geq 1,
$$

where $\zeta: \mathbb{N} \times \mathbb{Z} \rightarrow \mathbb{R}^{m \times m}$ is a kernel function satisfying the following conditions:

(i) $\zeta(n, k)$ is normalized so that $\zeta(n, s)=0$ for $s \geq-1$ and for $s \leq-d+1$ where $d>3$ is a positive integer;

(ii) There exists a positive real number $\gamma$ such that $\sup _{t \geq 0} \sum_{s=-d}^{0}\left\|\Delta_{s} \zeta(t, s)\right\| \leq \gamma$.

For any $a, b \in \mathbb{N}$, define $\mathbb{N}(a)=\{a, a+1, \ldots\}$ and $\mathbb{N}(a, b)=\{a, a+1, \ldots, b\}$ where $a \leq b$. By a solution of (4), we mean a sequence $x(n)$ of elements in $\mathbb{R}^{m}$ which is defined for all $n \in \mathbb{N}\left(n_{0}-d+1\right)$ and satisfies (4) for $n \in \mathbb{N}\left(n_{0}\right)$ for some $n_{0} \in \mathbb{N}$. It is easy to see that for any given $n_{0} \in \mathbb{N}$ and initial conditions of the form

$$
x(n)=\phi(n), \quad n \in \mathbb{N}\left(n_{0}-d+1, n_{0}+1\right),
$$

(4) has a unique solution $x(n)$ which is defined for $n \in \mathbb{N}\left(n_{0}-d+1\right)$ and satisfies the initial conditions (5). To emphasize the dependence of the solution on the initial point $n_{0}$ and the initial functions $\varphi$, we may use the notation $x(n)=x\left(n ; n_{0}, \varphi\right)$.

Our approach is based on constructing an adjoint equation for (4) with respect to a discrete analog for function (3) and proving that (4) and its adjoint equation have the same number of linearly independent periodic solutions. We shall employ some primary algebraic techniques to prove the main results of this article. It is worth mentioning here that the equation under consideration in this article (Equation (4)) is given in general form so it includes many particular cases of difference equations with pure delays; see [5,11-13] for more details.

\section{Preliminary assertions}

This section is devoted to certain auxiliary assertions that will be needed in the proof of the main theorem. Lemma 2.1 which introduces the main result of this section is needed to define an adjoint equation for (4). Lemmas 2.4 and 2.7 give representations of solutions of the considered equations. The proof of these lemmas were given in [14]. For the benefit of the readers, however, we state these lemmas along with their proofs. 
Consider the function

$$
<x(n), y(n)>=x^{T}(n) y(n)+\sum_{s=n-d}^{n} x^{T}(s-1) \Delta_{s} \sum_{\alpha=n+1}^{s+d-1} \zeta^{T}(\alpha, s-\alpha) y(\alpha),
$$

where $\Delta_{n} \zeta(n, k):=\zeta(n+1, k)-\zeta(n, k)$. We claim that the equation

$$
\Delta y(n)=-\Delta_{n} \sum_{k=-d}^{0} \zeta^{T}(n-k, k+1) y(n-k)
$$

is an adjoint equation of (4) with respect to (6). The following lemma proves meaningful.

Lemma 2.1 Let $x(n)$ be any solution of (4) and $y(n)$ be any solution of (7) then

$$
<x(n), y(n)>=\text { constant, }
$$

where $<\cdot, \cdot>$ is defined by (6).

Proof. Clearly, it suffices to show that $\Delta\langle x(n), y(n)\rangle=0$. It follows that

$$
\Delta<x(n), y(n)>=x^{T}(n) \Delta y(n)+\Delta x^{T}(n) y(n+1)+\Delta_{n} \sum_{s=n-d}^{n} g(s, n),
$$

where

$$
g(s, n)=x^{T}(s-1) \Delta_{s} \sum_{\alpha=n+1}^{s+d-1} \zeta^{T}(\alpha, s-\alpha) y(\alpha) .
$$

It is easy to see that

$$
\Delta_{n} \sum_{s=n-d}^{n} g(s, n)=g(n+1, n+1)-g(n-d, n)+\sum_{s=n-d+1}^{n} \Delta_{n} g(s, n) .
$$

Therefore (9) becomes

$$
\begin{aligned}
\Delta<x(n), y(n)>= & x^{T}(n) \Delta y(n)+\Delta x^{T}(n) y(n+1)+g(n+1, n+1) \\
& -g(n-d, n)+\sum_{s=n-d+1}^{n} \Delta_{n} g(s, n) .
\end{aligned}
$$

Thus

$$
\begin{aligned}
\Delta<x(n), y(n)>\stackrel{\operatorname{by}(7)}{=} x^{T}(n) & {\left[-\Delta_{n} \sum_{k=-d}^{0} \zeta^{T}(n-k, k+1) y(n-k)\right] } \\
& \stackrel{\operatorname{by}(4)}{+}\left[\sum_{k=-d}^{0} x^{T}(n+k-1) \Delta_{k} \zeta^{T}(n+1, k-1)\right] y(n+1) \\
& \stackrel{\operatorname{by}(10)}{+} x^{T}(n) \sum_{\alpha=n+3}^{n+d} \Delta_{n} \zeta^{T}(\alpha, n+1-\alpha) y(\alpha) \\
& \stackrel{\operatorname{by}(10)}{-} x^{T}(n-d-1) \Delta_{n-d} \sum_{\alpha=n+1}^{n-1} \zeta^{T}(\alpha, n-d-\alpha) y(\alpha) \\
& \stackrel{\operatorname{by}(10)}{-} \sum_{s=n-d+1}^{n} x^{T}(s-1) \Delta_{s} \zeta^{T}(n+1, s-n-1) y(n+1) .
\end{aligned}
$$


By changing the indices and using the properties of $\zeta$, we see that the above equation is equal to zero. The proof is finished.

Remark 2.2 In virtue of Lemma 2.1, we may say that Equation (7) is an adjoint of (4). It is easy to verify also that the adjoint of (7) is (4), i.e., they are mutually adjoint of each other.

Consider the nonhomogeneous equation

$$
\Delta x(n)=\sum_{k=-d}^{0} \Delta_{k} \zeta(n+1, k-1) x(n+k-1)+f(n), \quad n \geq 1,
$$

where $f$ is a sequence with values in $\mathbb{R}^{m}$.

Definition 2.3 A matrix solution $X(n, \alpha)$ of (4) satisfying $X(\alpha, \alpha)=I$, (I is an identity matrix), and $X(n, \alpha)=0$ for $n<\alpha$ is called a fundamental function of (4).

Lemma 2.4 Let $X(n, \alpha)$ be a fundamental function of (4) and $n_{0} \in \mathbb{N}$. If $x(n)$ is a solution of (11), then

$$
\begin{aligned}
x(n)= & X\left(n, n_{0}\right) x\left(n_{0}\right)+\sum_{s=n_{0}-d}^{n_{0}} \Delta_{s} \sum_{\alpha=n_{0}+1}^{s+d-1} X(n, \alpha) \zeta(\alpha, s-\alpha) x(s-1) \\
& +\sum_{k=n_{0}}^{n-1} X(n, k+1) f(k) .
\end{aligned}
$$

Proof. A direct substitution of (12) in (11) leads to the desired result. Indeed,

$$
\begin{aligned}
\Delta x(n)= & \Delta X\left(n, n_{0}\right) x\left(n_{0}\right)+\Delta_{n} \sum_{s=n_{0}-d}^{n_{0}} \Delta_{s} \sum_{\alpha=n_{0}+1}^{s+d-1} X(n, \alpha) \zeta(\alpha, s-\alpha) x(s-1) \\
& +\Delta_{n} \sum_{k=n_{0}}^{n-1} X(n, k+1) f(k)
\end{aligned}
$$

or

$$
\begin{aligned}
\Delta x(n) \stackrel{\text { by }(4)}{=} & \sum_{k=-d}^{0} \Delta_{k} \zeta(n+1, k-1) X\left(n+k-1, n_{0}\right) x\left(n_{0}\right) \\
& +\sum_{s=n_{0}-d}^{n_{0}}\left[\Delta_{s} \sum_{\alpha=n_{0}+1}^{s+d-1}\left\{\sum_{k=-d}^{0} \Delta_{k} \zeta(n+1, k-1) X(n+k-1, \alpha)\right\} \zeta(\alpha, s-\alpha)\right] x(s-1) \\
& +f(n)+\sum_{k=n_{0}}^{n-1}\left\{\sum_{k=-d}^{0} \Delta_{k} \zeta(n+1, k-1) X(n+k-1, k+1)\right\} f(k) \\
= & f(n)+\sum_{k=-d}^{0} \Delta_{k} \zeta(n+1, k-1) x(n+k-1) .
\end{aligned}
$$

Corollary 2.5 Let $X(n, \alpha)$ be a fundamental function of (4) and $n_{0} \in \mathbb{N}$. If $x(n)$ is a solution of (4), then

$$
x(n)=X\left(n, n_{0}\right) x\left(n_{0}\right)+\sum_{s=n_{0}-d}^{n_{0}} \Delta_{s} \sum_{\alpha=n_{0}+1}^{s+d-1} X(n, \alpha) \zeta(\alpha, s-\alpha) x(s-1) .
$$

Definition 2.6 A matrix solution $Y(n, \alpha)$ of $(7)$ satisfying $Y(\alpha, \alpha)=I$ and $Y(n, \alpha)=$ 0 for $n>\alpha$ is called a fundamental function of (7). 
Lemma 2.7 Let $Y(n, \alpha)$ be a fundamental function of $(7)$ and $n_{0} \in \mathbb{N}$. If $y(n)$ is a solution of (5), then

$$
y(n)=Y\left(n, n_{0}\right) y\left(n_{0}\right)+\sum_{s=n_{0}-d}^{n_{0}} Y(n, s-1) \Delta_{s} \sum_{\alpha=n_{0}+1}^{s+d-1} \zeta^{T}(\alpha, s-\alpha) \gamma(\alpha) .
$$

Corollary 2.8 Let $X\left(n ; n_{0}\right)$ be a fundamental function of $(4)$ and $Y\left(n, n_{0}\right)$ be a fundamental function of (7). Then

$$
X\left(n, n_{0}\right)=Y^{T}\left(n_{0}, n\right) .
$$

Proof. By following the same arguments used by Halanay in [[2], p. 364], (8) can be written as follows

$$
<x(n), y(n)>=<x\left(n_{0}\right), y\left(n_{0}\right)>, \quad \text { for any } n_{0} \in \mathbb{N} .
$$

Further

$$
\begin{aligned}
X^{T}(n, n) Y\left(n, n_{0}\right) & +\sum_{s=n-d}^{n} X^{T}(s-1, n) \Delta_{s} \sum_{\alpha=n+1}^{s+d-1} \zeta^{T}(\alpha, s-\alpha) Y\left(\alpha, n_{0}\right) \\
& \stackrel{\operatorname{by}(6)}{=} X^{T}\left(n_{0}, n\right) Y\left(n_{0}, n_{0}\right) \\
& +\sum_{s=n-d}^{n} X^{T}(s-1, n) \Delta_{s} \sum_{\alpha=n+1}^{s+d-1} \zeta^{T}(\alpha, s-\alpha) Y\left(\alpha, n_{0}\right) .
\end{aligned}
$$

Upon using the properties of the fundamental functions $X\left(n, n_{0}\right)$ and $Y\left(n, n_{0}\right)$, identity (16) is obtained.

Remark 2.9 Formulas (14) and (15) can be derived from function (6). Indeed, replacing $X$ by $x$ or $Y$ by $y$ in (17), using (16) and employing the properties of $X$ and $Y$ we obtain the desired results.

\section{The main results}

With regard to Equation (11), the following conditions are assumed to be valid throughout the remaining part of the article.

(i) $\zeta(n, k): \mathbb{N} \times \mathbb{Z} \rightarrow \mathbb{R}^{m \times m}$ is $p$ periodic sequence in $n, p>d$;

(ii) $f: \mathbb{N} \rightarrow \mathbb{R}^{m}$ is $p$ a periodic sequence, $p>d$.

Let $x(n)=x(n ; \phi)$ be the solution of Equation (11) defined for $n \geq 1$ such that $x(n)$ coincides with $\phi$ on $[-d+2,2]$. The periodicity of the equation implies that $x(n+p$; $\phi)$ is likewise a solution of the equation defined for $n+p \geq d$. If this solution coincides with $\phi$ in $[-d+2,2]$, then on the basis of the uniqueness theorem it follows that $x(n+$ $p ; \phi)=x(n ; \phi)$ for all $n \geq-d+2$ and the solution is periodic. Thus the periodicity condition of the solution is written as $x(n+p ; \phi)=\phi(n)$ for $n \in[-d+2,2]$. If $W$ is defined by $W \phi=x(n+p ; \phi), n \in[-d+2,2]$, then it follows that $x(n)$ is periodic if and only if $W \phi=\phi$, i.e., $\phi$ is a fixed point of $W$.

Let $z(n)=z(n ; \phi)$ be the solution of (4) defined for $n \geq 1$ such that $z(n)=\phi(n)$ on [- $d$ +2 , 2]. Then by Lemma 2.4 , 


$$
x(n ; \varphi)=z(n ; \varphi)+\sum_{k=0}^{n-1} X(n, k+1) f(k) .
$$

Define $U$ by $U \phi=z(n+p ; \phi), n \in[-d+2,2]$. Then, since

$$
W \varphi=U \varphi+\sum_{k=0}^{n+p-1} X(n+p, k+1) f(k)
$$

the periodicity condition reads as

$$
\varphi=U \varphi+\sum_{k=0}^{n+p-1} X(n+p, k+1) f(k) .
$$

Let $y(n)=y(n ; \psi)$ be the solution of (7) defined for $n \leq p+d$ such that $y(n)=\psi(n)$ on $[p, p+d]$. Similarly, we conclude that if $y(n-p ; \psi)$ coincides with $\psi$ in $[p, p+d]$ then $y(n-p ; \psi)=y(n ; \psi)$ and hence the solution is periodic. From Lemma 2.7, we get

$$
\psi(n)=X^{T}(p, n-p) \psi(p)+\sum_{s=p-d}^{p} X^{T}(s-1, n-p) \Delta_{s} \sum_{\alpha=p+1}^{s+d-1} \zeta^{T}(\alpha, s-\alpha) \psi(\alpha),
$$

for $n \in[p, p+d]$. Let $\tilde{\varphi}(s)=\psi(s+p+d)$ for $s \in[-d+2,2]$. Setting $\eta=k-p-d$, we find out

$$
\begin{aligned}
\tilde{\varphi}(s)= & X^{T}(p, s+d) \tilde{\varphi}(-d) \\
& +\sum_{s=p-d}^{p} X^{T}(s-1, s+d) \Delta_{s} \sum_{\eta=-d+1}^{s-p-1} \zeta^{T}(\eta+p+d, s-\eta-p-d) \tilde{\varphi}(\eta) .
\end{aligned}
$$

For sake of convenience, we also use the notation

$$
\begin{aligned}
<\Psi(s), \Phi(s)>= & \Psi^{T}(-d) \Phi(0) \\
& +\sum_{s=-d}^{0} \Delta_{s} \sum_{\eta=-d+1}^{s-p-1} \Psi^{T}(\eta) \zeta(\eta+p+d, s-\eta-p-d) \Phi(s)
\end{aligned}
$$

for matrix sequences $\Psi$ and $\Phi$ defined on $[-d+2,2]$ as long as multiplication is possible. Note that $<\Psi(s), \Phi(s)>$ could be either a number or a vector or a matrix, depending on the sizes of $\Psi$ and $\Phi$.

The following lemma, which is a discrete analogue of [4, Lemma 4], plays a key role in our later analysis. Its proof is straightforward and can be achieved directly by changing the order of summations.

Lemma 3.1 For any matrix sequences $N, M, L \in \mathbb{R}^{m \times m}$, we have

$$
<<(\sigma), M(\alpha, \sigma)>^{T}, N(\alpha)>=<L(\sigma),<M^{T}(\alpha, \sigma), N(\alpha)>>.
$$

By using this notation, the operator $U$ can be written as

$$
U \varphi=<X^{T}(p+s, \eta+d), \varphi(\eta)>.
$$


If we define $\tilde{U} \tilde{\varphi}=<\tilde{\varphi}(\eta), X(p+\eta, s+d)>^{T}$, then in view of Lemma 3.1 we obtain

$$
<\tilde{U} \tilde{\varphi}, \varphi>=<\tilde{\varphi}(\eta),<X^{T}(p+\eta, s+d), \varphi(s)>>=<\tilde{\varphi}, U \varphi>
$$

Let $\tilde{V} \psi=\gamma\left(n_{0}-p ; \psi\right)$ for $n_{0} \in[p, p+d]$. That is,

$$
\tilde{V} \psi=X^{T}\left(p, n_{0}-p\right) \psi(p)+\sum_{s=p-d}^{p} X^{T}\left(s-1, n_{0}-p\right) \Delta_{s} \sum_{\alpha=p+1}^{s+d-1} \zeta^{T}(\alpha, s-\alpha) \psi(\alpha)
$$

for $n_{0} \in[p, p+d]$. If $\rho$ is an eigenvalue of $\tilde{V}$, then there exists a nonzero solution of

$$
\begin{aligned}
\rho \tilde{\varphi}(s)= & X^{T}(p, s+d) \tilde{\varphi}(-d) \\
& +\sum_{s=p-d}^{p} X^{T}(s-1, s+d) \Delta_{s} \sum_{\eta=-d+1}^{s-p-1} \zeta^{T}(\eta+p+d, s-\eta-p-d) \tilde{\varphi}(\eta)
\end{aligned}
$$

where $\tilde{\varphi}(s)=\psi(s+p+d), s \in[-d+2,2]$. The right side of the above equation is nothing but $\tilde{U} \tilde{\varphi}$. Thus the eigenvalues of the operators $\tilde{U}$ and $\tilde{V}$ coincide and in addition, if $\psi$ is an eigenfunction for $\tilde{V}$, then $\tilde{\varphi}=\psi(s+p+d)$ is an eigenfunction for $\tilde{U}$.

Lemma 3.2 Equations (4) and (7) have the same number of linearly independent periodic solutions of period $p>d$.

Proof. Consider the equation

$$
\rho \varphi(s)-U \varphi(s)=F(s) .
$$

It is obvious that the fundamental function $X$ can be written as a linear combination of linearly independent vectors. That is,

$$
X(p+s, \xi+d)=\sum_{k=1}^{m} a_{k}(s) b_{k}(\xi)+K_{1}(s, \xi), \quad \text { for } s, \xi \in[-d+2,2] \times[-d+2,2],
$$

where $a_{k}(s)$ are column and $b_{k}(\xi)$ are row linearly independent vectors, $K_{1}$ is a matrix such that $\left|K_{1}\right|$ is chosen small. Clearly, we have

$$
X^{T}(p+s, \xi+d)=\sum_{k=1}^{m} b_{k}^{T}(\xi) a_{k}^{T}(s)+K_{1}^{T}(s, \xi)
$$

Then, by using the fact that $<b_{k}^{T}(\xi) a_{k}^{T}(s), \varphi(s)>=a_{k}(s)<b_{k}^{T}(\xi), \phi(s)>,(20)$ becomes

$$
\rho \varphi(s)-\sum_{k=1}^{m} a_{k}(s)<b_{k}^{T}(\xi), \varphi(\xi)>-<K_{1}^{T}(s, \xi), \varphi(\xi)>=F(s) .
$$

Setting

$$
\nu(s)=\frac{1}{\rho} \sum_{k=1}^{m} a_{k}(s)<b_{k}^{T}(\xi), \varphi(\xi)>+\frac{1}{\rho} F(s),
$$


we obtain

$$
v(s)=\varphi(s)-\frac{1}{\rho}<K_{1}^{T}(s, \xi), \varphi(\xi)>.
$$

Now consider equation of the form

$$
v(s)=\varphi(s)-\lambda<K_{1}^{T}(s, \xi), \varphi(\xi)>.
$$

We seek a solution of the form $\varphi(s)=\sum_{i=0}^{\infty} \lambda^{i} \varphi_{i}(s)$. Substituting this into (23) and identifying the coefficients of the powers of $\lambda$, we obtain

$$
\varphi_{0}(s)=v(s) \quad \text { and } \quad \varphi_{i}(s)=<K_{1}^{T}(s, \alpha), \varphi_{i-1}(\alpha)>, \quad i=1,2, \ldots
$$

It follows that $\left|\varphi_{i}(s)\right| \leq M^{i} \sup |v(s)|$, where $M=\sup \left|K_{1}^{T}\right|$ and $i=1,2, \ldots$ Therefore, the series converges if $|\lambda| M<1$. We have

$$
\varphi_{1}(s)=<K_{1}^{T}(s, \alpha), v(\alpha)>.
$$

By the induction principle, we obtain

$$
\varphi_{l}(s)=<K_{l}^{T}(s, \alpha), v(\alpha)>
$$

where $K_{l}(s, \xi)=<K_{1}^{T}(s, \alpha), K_{l-1}(\alpha, \xi)>$. Indeed, we have

$$
\varphi_{l+1}(s)=<K_{1}^{T}(s, \alpha), \varphi_{l}(\alpha)>=<K_{1}^{T}(s, \alpha),<K_{l}^{T}(\alpha, \xi), v(\xi)>>
$$

Using Lemma 3.1, we get

$$
\varphi_{l+1}(s)=<<K_{1}^{T}(s, \alpha), K_{l}(\alpha, \xi)>^{T}, v(\xi)>=<K_{l+1}^{T}(s, \xi), v(\xi)>
$$

It follows that, if $|\lambda|<\frac{1}{M}$ then the solution of Equation (23) can be written as

$$
\varphi(s)=v(s)+\sum_{l=1}^{\infty} \lambda^{l} \varphi_{l}(s)=v(s)+\sum_{l=1}^{\infty} \lambda^{l}<K_{l}^{T}(s, \alpha), v(\alpha)>
$$

Thus, $\phi(s)=v(s)+<\Gamma^{T}(s, \alpha), v(\alpha)>$ where $\Gamma^{T}(s, \alpha)=\sum_{l=1}^{\infty} \lambda^{l} K_{l}^{T}(s, \alpha)$. Therefore, if $\frac{1}{|\rho|}<\frac{1}{M}$ and $\sup \left|K_{1}^{T}\right|<|\rho|$, we deduce that

$$
\varphi(s)=v(s)+<\Gamma^{T}(s, \alpha), v(\alpha)>
$$

is a solution of (22).

On the other hand, consider the equation

$$
\rho \tilde{\varphi}(s)-\tilde{U} \tilde{\varphi}(s)=0,
$$

which can be written as

$$
\rho \tilde{\varphi}(s)=\sum_{k=1}^{m} b_{k}^{T}(s)<\tilde{\varphi}(\alpha), a_{k}(\alpha)>^{T}+<\tilde{\varphi}(\alpha), K_{1}(\alpha, s)>^{T} .
$$


Setting

$$
\tilde{v}(s)=\frac{1}{\rho} \sum_{k=1}^{m} b_{k}^{T}(s)<\tilde{\varphi}(\alpha), a_{k}(\alpha)>^{T},
$$

we obtain

$$
\tilde{v}(s)=\tilde{\varphi}(s)-\frac{1}{\rho}<\tilde{\varphi}(\alpha), K_{1}(\alpha, s)>^{T} .
$$

Following similar analysis, we obtain that the solution of (26) is in the form

$$
\tilde{\varphi}(s)=\tilde{v}(s)+<\tilde{v}(\alpha), \tilde{\Gamma}(\alpha, s)>^{T}
$$

where $\tilde{\Gamma}(\alpha, s)=\sum_{l=1}^{\infty} \lambda^{l} \tilde{K}_{l}(\alpha, s)$ and $\tilde{K}_{l}(\xi, s)=<K_{l-1}^{T}(\xi, \alpha), K_{1}(\alpha, s)>$. However, using the induction principle and Lemma 3.1 , it is easy to verify that $\tilde{K}_{l}(\xi, s)=K_{l}(\xi, s)$ by which one can see that

$$
\tilde{\Gamma}(\xi, s)=\Gamma(\xi, s) .
$$

In view of Equation (21), we have

$$
\rho v(s)=\sum_{k=1}^{m} a_{k}(s)<b_{k}^{T}(\xi), \varphi(\xi)>+F(s) .
$$

But $\phi(s)=v(s)+<\Gamma^{T}(s, \alpha), n(\alpha)>$. So

$$
\rho v(s)=\sum_{k=1}^{m} a_{k}(s)<b_{k}^{T}(\xi), v(\xi)+<\Gamma^{T}(\xi, \alpha), v(\alpha)>>+F(s),
$$

which can be written as

$$
\rho v(s)=\sum_{k=1}^{m} a_{k}(s)\left(<b_{k}^{T}(\xi), v(\xi)>+<b_{k}^{T}(\xi),<\Gamma^{T}(\xi, \alpha), v(\alpha)>>\right)+F(s) .
$$

Using Lemma 3.1, we get

$$
\rho v(s)=\sum_{k=1}^{m} a_{k}(s)<b_{k}^{T}(\alpha)+<b_{k}^{T}(\xi), \Gamma(\xi, \alpha)>^{T}, v(\alpha)>+F(s) .
$$

Hence

$$
\rho v(s)=\sum_{k=1}^{m} a_{k}(s)<\bar{b}_{k}^{T}(\alpha), v(\alpha)>+F(s),
$$

where $\bar{b}_{k}^{T}(\alpha)=b_{k}^{T}(\alpha)+<b_{k}^{T}(\xi), \Gamma(\xi, \alpha)>^{T}$. Setting $\lambda_{k}=<\bar{b}_{k}^{T}(\alpha), v(\alpha)>$, it follows from (30) that

$$
\rho v(s)-F(s)=\sum_{k=1}^{m} \lambda_{k} a_{k}(s)
$$


is the form of the solution of (30). Analogously, the solution of

$$
\rho \tilde{v}(s)=\sum_{k=1}^{m} b_{k}^{T}(s)<\tilde{v}(\xi), \bar{a}_{k}(\xi)>^{T},
$$

has the form

$$
\rho \tilde{v}(s)=\sum_{k=1}^{m} \mu_{k} b_{k}^{T}(s)
$$

where $\mu_{k}=<\tilde{v}(\xi), \bar{a}_{k}(\xi)>^{T}$ and $\bar{a}_{k}(\xi)=a_{k}(\xi)+<\tilde{\Gamma}^{T}(\xi, \alpha), a_{k}(\alpha)>$. In view of (30), (31)

becomes

$$
\sum_{k=1}^{m} \lambda_{k} a_{k}(s)=\sum_{k=1}^{m} a_{k}(s)<\bar{b}_{k}^{T}(\alpha), \frac{1}{\rho} F(\alpha)+\frac{1}{\rho} \sum_{j=1}^{m} \lambda_{j} a_{j}(\alpha)>.
$$

Similarly, Equation (32) implies that (33) can be written as

$$
\sum_{k=1}^{m} \mu_{k} b_{k}^{T}(s)=\sum_{k=1}^{m} b_{k}^{T}(s)<\frac{1}{\rho} \sum_{j=1}^{m} \mu_{j} b_{j}^{T}(\xi), \bar{a}_{k}(\xi)>^{T} .
$$

Taking into account that the vectors $\left\{a_{k}\right\}$ are linearly independent, we obtain from (34) the algebraic equation

$$
\rho \lambda_{k}=\sum_{j=1}^{m} \gamma_{k j} \lambda_{j}+f_{k}
$$

where $\gamma_{k j}=<\bar{b}_{k}^{T}(\alpha), a_{j}(\alpha)>$ and $f_{k}=<\bar{b}_{k}^{T}(\alpha), F(\alpha)>$. Similarly, we get from (35) the algebraic equation

$$
\rho \mu_{k}=\sum_{j=1}^{m} \tilde{\gamma}_{j k}^{T} \mu_{j}
$$

where $\tilde{\gamma}_{j k}^{T}=<b_{j}^{T}(\xi), \bar{a}_{k}(\xi)>$. We know that Equation (36) for $\lambda_{k}$ has a solution if and only if

$$
\sum_{k=1}^{m} \mu_{k} f_{k}=0
$$

for all the solutions $\mu_{k}$ of the equation

$$
\rho \mu_{k}=\sum_{j=1}^{m} \gamma_{j k} \mu_{j}
$$

By employing Lemma 3.1 and relation (28), however, we can obtain that $\tilde{\gamma}_{j k}^{T}=\gamma_{j k}$. Thus, Equations (37) and (39) coincide. 
Therefore, we conclude that the equations

$$
\rho \lambda_{k}=\sum_{j=1}^{m} \gamma_{k j} \lambda_{j}
$$

and

$$
\rho \mu_{k}=\sum_{j=1}^{m} \gamma_{j k} \mu_{j}
$$

have the same number of linearly independent solutions. To a solution of (40) corresponds $v(s)=\frac{1}{\rho} \sum_{k=1}^{m} \lambda_{k} a_{k}(s)$ and to this corresponds the solution $\phi(s)=v(s)+<\Gamma^{T}$ $(s, \alpha), v(\alpha)>$ for the equation $\rho \phi(s)-U \phi(s)=0$, linearly independent solutions corresponding to the linearly independent solutions of Equation (40). Likewisely, a solution of the equation $\rho \tilde{\varphi}(s)-\tilde{U} \tilde{\varphi}(s)=0$ will correspond to a solution of Equation (37) which coincides with (41), linearly independent solutions corresponding to linearly independent solutions. It follows from here that the equations $\rho \phi(s)-U \phi(s)=0$ and $\rho \tilde{\varphi}(s)-\tilde{U} \tilde{\varphi}(s)=0$ have the same number of independent solutions, which implies in particular the fact that $U$ and $\tilde{U}$ have the same eigenvalues, hence if $\rho$ is a multiplier of the equation, $\frac{1}{\rho}$ is a multiplier of the adjoint equation. The proof of Lemma 3.2 is completed.

We are now in a position to state and prove the main result of this article.

Theorem 3.3 A necessary and sufficient condition for the existence of periodic solutions of period $p$ of Equation (11) is that

$$
\sum_{k=0}^{p-1} y^{T}(k+1) f(k)=0,
$$

for all periodic solutions $y(n)$ of period $p$ of the adjoint Equation (7).

NECESSITY. Let $x(n)$ be $p$ periodic solution of (11) and $y(n) p$ periodic solution of (7). It follows that $<y(n), x(n)>$ is $p$ periodic. In view of (7) and (11), one can conclude that

$$
\Delta<\gamma(n), x(n)>=\gamma^{T}(n+1) f(n), \quad 0 \leq n \leq p .
$$

Summing (43) over the interval $[0, p-1]$ results in

$$
\sum_{k=0}^{p-1} y^{T}(k+1) f(k)=0,
$$

which is the same as (42).

SUFFICIENCY. Suppose that (42) is satisfied for all periodic solutions $y(n)$ of period $p$ of (7). In virtue of relation (38), Lemma 3.2 tells us that

$$
\rho \varphi(s)-U \varphi(s)=F(s)
$$

has solutions if and only if

$$
<\tilde{\varphi}(\alpha), F(\alpha)>=0
$$


for all $\tilde{\varphi}$ satisfying

$$
\rho \tilde{\varphi}(s)-\tilde{U} \tilde{\varphi}(s)=0 .
$$

Therefore, it suffices to show that (44) holds under condition (42). We observe from (18) that

$$
F(s)=\varphi(s)-U \varphi(s)=\sum_{k=0}^{s+p-1} X(s+p, k+1) f(k) .
$$

It follows that

$$
\begin{aligned}
<\tilde{\varphi}(\alpha), F(\alpha)>= & \tilde{\varphi}^{T}(-d) F(0) \\
& +\sum_{s=-d}^{0} \Delta_{s} \sum_{\eta=-d+1}^{s-p-1} \tilde{\varphi}^{T}(\eta) \zeta(\eta+d, s-\eta-p-d) F(s) .
\end{aligned}
$$

Substituting $F$ into (45) leads to

$$
\begin{aligned}
<\tilde{\varphi}(\alpha), F(\alpha)>= & \tilde{\varphi}^{T}(-d) \sum_{k=0}^{p-1} X(p, k+1) f(k) \\
& +\sum_{s=-d}^{0} \Delta_{s} h(s)\left[\sum_{r=0}^{k+p-1} X(p+k, r+1) f(r)\right],
\end{aligned}
$$

where $h(s)=\sum_{\eta=-d+1}^{s-p-1} \tilde{\varphi}(\eta) \zeta(\eta+d, s-\eta-p-d)$. Setting $\tilde{\varphi}(s)=\psi(s+p+d)$ and interchanging the order of summations, we obtain

$$
\begin{aligned}
<\tilde{\varphi}(\alpha), F(\alpha)>= & \psi^{T}(p) \sum_{k=0}^{p-1} X(p, k+1) f(k) \\
& +\sum_{r=0}^{p-1} \sum_{s=p-d}^{p} \Delta_{s} \sum_{\alpha=p+1}^{s+d-1} \psi^{T}(\alpha) \zeta(\alpha, s-\alpha) X(s-1, k+1) f(k) .
\end{aligned}
$$

Reordering the terms, we end up with

$$
\begin{aligned}
<\tilde{\varphi}(\alpha), F(\alpha)>= & \sum_{k=0}^{p-1}\left[\psi^{T}(p) X(p, k+1)\right. \\
& \left.+\sum_{s=p-d}^{p} \Delta_{s} \sum_{\alpha=p+1}^{s+d-1} \psi^{T}(\alpha) \zeta(\alpha, s-\alpha) X(s-1, k+1)\right] f(k) .
\end{aligned}
$$

In view of Lemma 2.7 we see that the right hand side of the above equation is nothing but

$$
\sum_{k=0}^{p-1} y^{T}(k+1) f(k)
$$

which is clearly zero by our assumption (42). The proof is finished.

Example 1 Equations (4) and (7) can be reduced to the following difference equations with pure delays 


$$
\Delta x(n)=A(n) x(n)+B(n+1) x(n-j+1), \quad n \geq 1
$$

and

$$
\Delta y(n)=-A^{T}(n) y(n+1)-B^{T}(n+j) y(n+j)
$$

where $2<j$ is a fixed positive integer number and $A, B: \mathbb{N} \rightarrow \mathbb{R}^{m \times m}$ are p periodic sequences, $p>j$. In virtue of [5, Lemma 2], we find that $\langle y(n), x(n)\rangle=$ constant, where

$$
<y(n), x(n)>=\gamma^{T}(n) x(n)+\sum_{k=n+1}^{n+j-1} y^{T}(k) B(k) x(k-j) .
$$

Of particular cases, we take $A(n)=3, B(n)=5, j=3$ and $f(n)=\cos \frac{n \pi}{2}$. Then, the equations

$$
\Delta x(n)=3 x(n)+5 x(n-2)+\cos \frac{n \pi}{2}, \quad n \geq 1
$$

and

$$
\Delta y(n)=-3 \gamma(n+1)-5 y(n+3)
$$

are mutually adjoint to each other with respect to the function

$$
<y(n), x(n)>=\gamma(n) x(n)+5 \sum_{k=n+1}^{n+2} y(k) x(k-3)
$$

One can easily see that $\cos \frac{n \pi}{2}$ is periodic of period 4 so $p=4>3$. It follows that the condition (42) becomes

$$
\sum_{k=0}^{3} y(k+1) \cos \frac{n \pi}{2}=y(1)-\gamma(3)
$$

which is equal to zero for any periodic solution y of Equation (50) under the initial condition $y(1)-y(3)=0$. By the result of Theorem 3:3, we conclude that there exist periodic solutions of period 4 for Equation (49).

\section{Acknowledgements}

The author would like to express his sincere thanks for the valuable comments of the reviewers which improved the exposition of the article.

\section{Competing interests}

The author declares that they have no competing interests.

Received: 8 February 2012 Accepted: 3 May 2012 Published: 3 May 2012

\section{References}

1. Miller, RK, Michal, AN: Ordinary Differential Equations. Academic Press, New York (1982)

2. Halanay, A: Differential Equations: Stability, Oscillation, Time Lags. Academic Press, New York (1966)

3. Alzabut, JO: A necessary and sufficient condition for the existence of periodic solutions of linear impulsive differential equations with distributed delay. Discr Contin Dyn Syst Supplement. 35-43 (2007)

4. Akhmet, MU, Alzabut, JO, Zafer, A: On periodic solutions of linear impulsive delay differential systems. Dyn Contin Discr Impuls Syst Ser A Math Anal. 15(5):621-631 (2008)

5. Alzabut, JO: On existence of periodic solutions for a class of linear delay difference equations. Commun Appl Anal. 14(2):191-202 (2010) 
6. Dannan, F, Elaydi, S, Liu, P: Periodic solutions of difference equations. J Diff Equ Appl. 6(2):203-232 (2000). doi:10.1080/ 10236190008808222

7. Elaydi, S, Zhang, S: Stability and periodicity of difference equations with finite delay. Funkcial Ekvac. 37(3):401-413 (1994)

8. Cai, $X, Y u, J$ : Existence theorems of periodic solutions for second-order nonlinear difference equations. Adv Diff Equ 11 (2008). (Article ID 247071) (2008). doi:10.1155/2008/247071

9. Vidal, C: Existence of periodic and almost periodic solutions of abstract retarded functional difference equations in phase spaces. Adv Diff Equ 19 (2009). (Article ID 380568) (2009). doi:10.1155/2009/380568

10. Song, Y: Periodic and almost periodic solutions of functional difference equations with finite delay. Adv Diff Equ 15 (2007). (Article ID 68023)(2007). doi:10.1155/2007/68023

11. Agarwal, RP, Wong, PJY: Advanced Topics in Difference Equations. Kluwer, Dordrecht. (1997)

12. Agarwal, RP: Difference Equations and Inequalities, Theory, Methods and Applications. Marcel Dekker, New York, 2 (2000)

13. Elayadi, S: An Introduction to Difference Equations. Springer, New York, 3 (2005)

14. Alzabut, JO, Abdeljawad, T: Perron-type criterion for linear difference equations with distributed delay. Discr Dyn Natl Soc 12 (2007). (Article ID 10840) (2007). doi:10.1155/2007/10840

doi:10.1186/1687-1847-2012-53

Cite this article as: Alzabut: Existence of periodic solutions for a type of linear difference equations with distributed delay. Advances in Difference Equations 2012 2012:53.

\section{Submit your manuscript to a SpringerOpen ${ }^{\circ}$} journal and benefit from:

- Convenient online submission

- Rigorous peer review

- Immediate publication on acceptance

- Open access: articles freely available online

- High visibility within the field

- Retaining the copyright to your article

Submit your next manuscript at $\gg$ springeropen.com 